\title{
Attitudes of pregnant women and male partners towards non-invasive prenatal testing and widening the scope of prenatal screening
}

\author{
Rachèl V van Schendel ${ }^{\star, 1}$, Johanna H Kleinveld ${ }^{1}$, Wybo J Dondorp ${ }^{2}$, Eva Pajkrt ${ }^{3}$, Danielle RM Timmermans ${ }^{4}$, \\ Kim CA Holtkamp ${ }^{1}$, Margreet Karsten ${ }^{1}$, Anne L Vlietstra ${ }^{1}$, Augusta MA Lachmeijer ${ }^{5}$ and Lidewij Henneman ${ }^{1}$
}

Non-invasive prenatal testing (NIPT) and its potential to test for multiple disorders has received much attention. This study explores attitudes of women and men towards NIPT, and their views on widening the scope of prenatal testing in a country with a low uptake of prenatal screening (The Netherlands). Five focus groups with low-risk pregnant women $(n=28)$, three focus groups with men $(n=19)$ and 13 interviews with high- and low-risk pregnant women were conducted. Participants felt that current prenatal screening has great disadvantages such as uncertain results and risk of miscarriage from follow-up diagnostics. Characteristics of NIPT (accurate, safe and early testing) could therefore diminish these disadvantages of prenatal screening and help lower the barrier for participation. This suggests that NIPT might allow couples to decide about prenatal testing based mostly on their will to test or not, rather than largely based on fear of miscarriage risk or the uncertainty of results. The lower barrier for participation was also seen as a downside that could lead to uncritical use or pressure to test. Widening the scope of prenatal testing was seen as beneficial for severe disorders, although it was perceived difficult to determine where to draw the line. Participants argued that there should be a limit to the scope of NIPT, avoiding testing for minor abnormalities. The findings suggest that NIPT could enable more meaningful decision-making for prenatal screening. However, to ensure voluntary participation, especially when testing for multiple disorders, safeguards on the basis of informed decision-making will be of utmost importance.

European Journal of Human Genetics (2014) 22, 1345-1350; doi:10.1038/ejhg.2014.32; published online 19 March 2014

\section{INTRODUCTION}

Non-invasive prenatal testing (NIPT) allows detection of chromosomal aneuploidies in the foetus by using circulating cell-free foetal DNA (cffDNA) in the plasma of pregnant women. ${ }^{1}$ This technique makes it possible to detect foetal trisomies 21,13 and 18 as early as 9 weeks into the pregnancy, and perhaps even earlier. ${ }^{2}$ As the procedure of NIPT consists of only drawing a blood sample, it eliminates the risk of miscarriage associated with invasive diagnostic procedures, that is, chorionic villus sampling (CVS) and amniocentesis. Consequently, NIPT assures both earlier and safer prenatal testing, although diagnostic confirmation of abnormal NIPT results by invasive testing is still required due to the chance of false positive test results. ${ }^{3}$

In 2010, Lo et $a l^{4}$ showed that it is possible to deduce the entire genomic sequence of a foetus through NIPT, implying that NIPT could identify a much wider range of genetic disorders in the future. These major developments in the field of prenatal testing are generating a great amount of debate regarding the potential impact, benefits and drawbacks of NIPT. Greely ${ }^{5}$, for example, provides a summary of the ethical questions that have been raised with regard to the implications of NIPT on the current prenatal screening setting and even on society as a whole. ${ }^{5}$ Where many women now decline prenatal screening due to the miscarriage risk of follow-up diagnostic testing, ${ }^{6}$ it is likely that more women will consider NIPT as it does not jeopardise the health of their foetus. Ethicists, however, worry that the safety and ease of the test might lead to normalisation of testing (trivial to offer and take) and to a decrease in informed choice, as parents might not be aware or fully comprehend what they are consenting to. ${ }^{5,7}$ Other concerns that are being raised include whether earlier testing might lead to trivialisation of selective abortions, ${ }^{7}$ or that the future prospective of broadening the scope of prenatal testing might lead to identifying and possibly aborting foetuses affected with minor abnormalities or non-medical traits. ${ }^{8}$ It has also been argued that broadening the scope of NIPT could seriously complicate informed consent, counselling and decision-making. ${ }^{7}$

As pregnant women are the target group for testing, it is important to explore their views and potential concerns on NIPT. In general, it has been shown that there is high (hypothetical) interest in NIPT among pregnant women. ${ }^{9}$ However, certain concerns, such as a potential increase of abortion rates with the introduction of NIPT, have been expressed by women. ${ }^{10}$ A recent study in the UK by Lewis et $a l^{11}$ also showed that women have concerns about the test

\footnotetext{
${ }^{1}$ Department of Clinical Genetics, Section of Community Genetics, EMGO Institute for Health and Care Research, VU University Medical Center, Amsterdam, The Netherlands; 2Department of Health, Ethics and Society, Faculty of Health, Medicine and Life Sciences, Research Institutes GROW and CAPHRI, Maastricht University, Maastricht, The Netherlands; ${ }^{3}$ Department of Obstetrics and Gynaecology, Fetal Medicine Unit, Academic Medical Centre, Amsterdam, The Netherlands; ${ }^{4}$ Department of Public and Occupational Health, EMGO Institute for Health and Care Research, VU University Medical Center, Amsterdam, The Netherlands; ${ }^{5}$ Department of Clinical Genetics, VU University Medical Center, Amsterdam, The Netherlands

*Correspondence: Ms RV van Schendel, Department of Clinical Genetics, Section of Community Genetics, EMGO Institute for Health and Care Research, VU University Medical Center, PO Box 7057 (BS7 D450), 1007 MB Amsterdam, The Netherlands. Tel: +31 20444 8914; Fax: +31 20 444 8665; E-mail: r.schendel@vumc.nl
}

Received 19 November 2013; revised 31 January 2014; accepted 31 January 2014; published online 19 March 2014 
becoming routinised and that pressure to test will increase as women might feel less justified to decline a test that is so simple and free of risk. In addition, a discrepancy was found between the attitudes of healthcare professionals and women. Women seem to place more value on the safety of the test and are prepared to wait longer and accept lower accuracy if the test has no risk of miscarriage, whereas healthcare professionals prefer a test that is more accurate. $^{12}$

The aim of this study is to further explore women's attitudes and concerns about NIPT and explore their views on widening the scope of prenatal screening through NIPT. Male partners are often not included in research but as decisions about prenatal testing are preferably made by both partners, and research has shown that men want to be involved in this decision-making process, ${ }^{13}$ the views of male partners were also explored in this study. Most studies about pregnant women's attitudes towards NIPT have been based on quantitative methods, ${ }^{9,10,12,14}$ which have the advantage of being able to include a large study sample. However, they lack a thorough, in-depth analysis of these attitudes. In this study, we therefore opted for qualitative research methods. The study has been conducted in The Netherlands, which offers a unique setting as the uptake of the first trimester combined screening test for trisomies (T21, 13 and 18) is low $(\sim 27 \%),{ }^{15}$ compared with, for example, Denmark or France where the uptake is around $90 \% .^{16,17}$ It is therefore interesting to see how the attitudes of pregnant women and partners on NIPT relate to the apparent reluctance regarding the combined test in The Netherlands.

\section{MATERIALS AND METHODS}

A qualitative study design was used: focus groups and individual semistructured interviews were carried out between May 2012 and May 2013. Focus groups were chosen as they trigger lively discussions and are designed to explore multiple perspectives. ${ }^{18}$ Interviews were held to allow the studying of the depth of women's attitudes and experiences but also to create a more private environment for women with a high-risk screening result to share their, often emotional, experiences with prenatal screening. Ethical approval for this study was granted by the Medical Ethical Committee of the VU University Medical Center Amsterdam.

\section{Focus groups}

Five homogeneous focus groups were conducted with pregnant women (invited $>22$ weeks gestation) at low risk of having a child with Down syndrome. 'Low-risk' was defined as women who obtained a low-risk result after the first trimester combined test $(>1: 200)$ or those who had decided to refrain from doing this test. Participants were recruited through midwifery practices and yoga schools for pregnant women in Amsterdam and a smaller city near Amsterdam. In total, 28 pregnant women were willing to participate. The response rate is unknown as recruitment was done by third parties. The focus groups were held at one midwifery practice $(n=3)$ and in two different community centres $(n=2)$. After five focus groups saturation had been reached. Participant characteristics are summarised in Table 1.

Online focus groups were used to explore the attitudes of male partners (invited $>22$ weeks gestation). We expected this methodology to facilitate recruitment as more men would be willing to participate when they are able to join the discussions at their own convenience, as has been shown among study populations in other domains. ${ }^{19}$ In addition to approaching several midwifery practices, men were recruited by placing a link to an invitation letter on 20 different websites and other social media platforms for parents-to-be. In total, 19 men were willing to participate in the study (Table 1). Two men were partners of women who had previously participated in the focus groups. Also here, the response rate is unknown as recruitment was done by third parties and through web pages.
Table 1 Characteristics of the female and male participants in the focus groups and individual interviews

\begin{tabular}{|c|c|c|c|c|}
\hline & \multicolumn{2}{|c|}{ Focus groups } & \multicolumn{2}{|c|}{ Individual interviews } \\
\hline & & & Low-risk & High-risk \\
\hline & Women & Men & women & women \\
\hline & $(n=28)$ & $(n=19)$ & $(n=6)$ & $(n=7)$ \\
\hline Mean age, years (range) & $\begin{array}{c}29.4 \\
(21-38)\end{array}$ & $\begin{array}{c}36.2 \\
(27-49)\end{array}$ & $\begin{array}{c}32.5 \\
(30-36)\end{array}$ & $\begin{array}{c}35.3 \\
(28-40)\end{array}$ \\
\hline
\end{tabular}

Weeks of gestation

$\begin{array}{lrrrr}<22 & 0 & 5 & 0 & 2 \\ \geq 22 & 28 & 12 & 6 & 4 \\ \text { Just delivered } & 0 & 2 & 0 & 1\end{array}$

Level of education ${ }^{\text {a }}$

$\begin{array}{lrrrr}\text { Low } & 4 & 0 & 0 & 0 \\ \text { medium } & 4 & 3 & 0 & 1 \\ \text { High } & 20 & 16 & 6 & 6\end{array}$

Ethnicity

Dutch

Other Western

Non-Western

17

Children

Yes

15

No

Religion

None

Calvinist/reformed

(Roman) Catholic

Muslim

Jewish

Missing

5

6

17

1

5

7

1

0

0

First trimester combined test

Yes

No

12
16

9

10

2

4

7

20-week ultrasound/anomaly scan

Yes

28

No

0

17

2

6

0

6

aLow: elementary school, lower level of secondary school, lower vocational training; Medium: higher level of secondary school, intermediate vocational training; and High: higher vocational training university.

${ }^{b}$ Ethnicity was coded as Dutch, other Western or non-Western by the following algorithm: by country of birth if not The Netherlands, if The Netherlands then by country of birth of mother, if the subject and mother were both born in The Netherlands then by country of father.

\section{Interviews}

Individual semi-structured interviews were conducted with six other pregnant women at low risk and seven women at high risk of having a child with Down syndrome. 'High-risk' was defined as women who had obtained a high-risk result after the first trimester combined test $(\leq 1: 200)$. Low-risk women were recruited through midwifery practices. A gynaecologist working at the Fetal Medicine Unit of the Academic Medical Center in Amsterdam selected and recruited high-risk women with different types of experiences. Five out of seven high-risk women had undergone invasive testing (amniocentesis or CVS) four of whom had received a normal result and one a diagnosis for Down syndrome, which was followed by the termination of the pregnancy. Two women had refrained from having invasive diagnosis; one of whom had chosen to have NIPT (abroad) and had received a normal test result. After 13 interviews, saturation had been reached and no further interviews were conducted. 


\section{Methodology}

The focus group sessions were conducted using a semi-structured interview protocol which comprised the following topics: participant's perceptions on the current Down syndrome screening, perceptions on the advantages and disadvantages of NIPT compared with the current Down syndrome screening, the consequences of testing for a wider range of diseases and information preferences. For the focus groups with women, a brief description of NIPT was first given using a PowerPoint presentation which further explained the steps in the current screening process and test characteristics of NIPT. Participants were told that it is expected that more genetic disorders could be tested in the future using NIPT. The focus groups were managed by an experienced moderator, with two assistants taking notes and observing the group interactions. For the individual interviews, the same semi-structured interview protocol was used, but high-risk women were asked some additional questions about their personal experiences and decision-making regarding their high-risk screening result.

The online focus groups with men were conducted through an internet forum (facilitated by The Netherlands Organisation for Applied Scientific Research: TNO). Participants were given a unique password for anonymous access. The first two focus groups were organised asynchronously; for 1 week, a new topic was posted on the forum daily, and men could participate at their convenience. A third focus group was organised synchronously, implying that participants were simultaneously online and asked to answer and respond to the questions posted at a pre-arranged time for $90 \mathrm{~min}$. The moderator supervised the discussions by using unscripted probes.

\section{Analysis}

Interviews with women were audiotaped and transcribed verbatim. For the focus groups with male partners, responses were copied from the online forum. Content analysis was performed using the qualitative software program ATLAS.ti 5.2. Analysis started with a transcript being read in detail, after which codes were created for recurring topics in the text. Codes were ranked and clustered into main topics and subtopics. To ensure uniform coding, analysis was performed independently by two researchers. Coded transcripts were compared between researchers, and discrepancies were discussed until consensus was reached. ${ }^{20}$ Representative quotes from the focus groups and interviews were translated from Dutch and are presented to illustrate the findings.

\section{RESULTS}

When asked about their attitudes towards NIPT in comparison with the current prenatal screening tests, and their views on widening the scope of prenatal screening through NIPT, participants expressed a variety of opinions and concerns. Views of women and men are described in detail below. Overall, no striking differences were observed in attitudes between men and women.

\section{Current prenatal screening for Down syndrome}

In general, participants were quite positive about having the option of prenatal screening for foetal trisomies. It became clear, however, that participants felt that the current prenatal screening has a number of major disadvantages. Participants mentioned that the combined test only gives a risk estimation, which is difficult to comprehend and does not give much certainty.

I think that the combined test is not so popular because it only gives you a risk-estimation and therefore it sometimes causes anxiety and worries.' (\#65, man)

Moreover, the fact that a potential follow-up test carries a small risk of miscarriage was seen as an important reason for people not to participate in prenatal screening. Some high-risk women also mentioned that when they, despite the risk of miscarriage, made the decision for invasive diagnosis, the procedure itself and the period after caused a lot of anxiety.

'I did feel scared that it would go wrong. For two weeks after the invasive procedure you still have a considerable chance that it goes wrong [miscarriage] so you are not relaxed. [...] It was all very stressful to me.'(\#41, high-risk woman)

In The Netherlands, the combined test is not covered by health insurance for women younger than 36 years. According to the participants, this also creates a barrier for participation as not everyone is financially able or willing to pay for the test (which costs $\sim 150$ euros).

\section{NIPT versus the combined test}

The initial attitude of participants towards NIPT was very positive. All participants agreed that the increased accuracy of NIPT was a great advantage.

'This test gives much more certainty than the combination of the ultrasound and blood test, which gives quite some room for speculation, and it's... well the combined test is of course also not invasive but personally I would have a much better feeling with this [NIPT] test' (\#38, high-risk woman)

Moreover, participants thought it was an important advantage that NIPT reduces the chance of needing to continue with a (often unnecessary) follow-up invasive test. This was seen as a great improvement as they believed many people nowadays do not wish to participate in prenatal screening or do not want to continue with follow-up testing due to the fear of a miscarriage caused by the procedure. Another aspect of NIPT that participants were positive about was the fact that this test can be performed earlier in pregnancy compared with the combined test. The participants hypothesised that testing, and potentially terminating the pregnancy, at an earlier stage is easier because of a less-intense emotional bond between mother and her unborn child.

'Oh yes, get it over with, you know. And yes, well I may not be religious, but I do believe that at one moment in time, this child will have a soul. So yes, the older it is, the more of a bond you will have. So if you want to terminate it, it's better to do this early in the pregnancy' (\#36, low-risk woman)

Moreover, the participants thought that earlier testing would mean that parents are left in doubt for a shorter period of time and in the case of a negative result can enjoy a 'care-free' pregnancy for a longer period of time.

Participants agreed that the characteristics of NIPT, that is, the increased accuracy, lower chance of needing follow-up invasive testing and earlier performance take away the disadvantages that couples experience with the current prenatal tests. NIPT could therefore lower the barrier for participation and this was seen as a great benefit by participants. Consequently, participants expected the uptake of prenatal screening to increase as more people, who now decline screening, are likely to accept screening with NIPT. Some of the participants, especially high-risk women, also expressed a personal interest in NIPT. However, participants thought that some people would still decline screening due to factors not associated with the test 
characteristics, for example, having a positive attitude towards having a child with Down syndrome.

'Undoubtedly, more women will now [with NIPT] have prenatal screening. The big uncertainty of the combined test and the chance of having an unnecessary follow-up test work as a barrier for many women. The high certainty of this new test [NIPT] will therefore lower the barrier for having prenatal screening' (\#16, man)

\section{NIPT: the other side of the coin}

Although participants were enthusiastic about NIPT at first, when they were asked to think about its potential disadvantages, a number of concerns arose. According to the participants, certain aspects of NIPT, which they initially considered as advantages, could potentially also create problems. The participants mentioned that the ease and lack of risk of NIPT could lead to women feeling less justified to saying no to this test, as there is basically no 'excuse' to decline it anymore. Some participants feared that because of earlier testing in pregnancy, pregnant couples could make a less-informed decision as they have had less time to read and digest information about this test, consider all the possible scenarios, and think about whether they wish to test or not.

'A disadvantage may be that you should take in all the information about the test and the potential result of it earlier than otherwise, which could be a challenge for the counselling. You may not even have visited the midwife yet and then you are already being offered all these possibilities' (\#53, man)

Furthermore, participants worried that as NIPT can be performed so early, this could create a problem for women who receive an abnormal result. They argued that these women need invasive follow-up testing for confirmation of the NIPT result, but this cannot be done straight away. Therefore, there will be a rather big time-gap between NIPT and the follow-up invasive test, during which the women could experience a great amount of uncertainty.

'You have the test [NIPT] at 9 weeks and chorionic villus sampling can only be performed from 11 weeks and amniocentesis from 15 weeks? Then there is a longer period in between in which you feel so uncertain' (\#27, low-risk woman)

Where previously participants had perceived the relatively low barrier for use of NIPT as an advantage, it became clear to them that it could also have a downside. Some participants thought that it could lead to women agreeing to this test without thoroughly thinking through this decision.

Maybe people will then more easily think 'I'm just going to do it [NIPT]', without really thinking thoroughly about it like 'What am I about to do?' and 'Then I will get a result but do I really want that?' (\#37, low-risk woman)

They also worried that as NIPT is a blood test and women already have many blood samples drawn during pregnancy that NIPT would just become another pregnancy routine and that could also lead to women not thoroughly thinking through the decision.

'Because it [NIPT] is a blood test and you have to go get blood drawn anyway and then maybe you think let's tick the box on the form for doing this blood test right away as well.' (\#23, low-risk woman)
Moreover, some participants feared that when NIPT becomes widely used (because of the easier accessibility), fewer disabled children would be born and as a consequence parents who decide not to test or decide to keep a child with, for example, Down syndrome, will become stigmatised. Some participants also expressed a concern that there would be little acceptance but also less support for children with Down syndrome if more people were to use NIPT. This could lead to women feeling obliged to test as having a child with Down syndrome is not really feasible anymore.

'Yes it's an interaction because the more we test, the less there will be [children with Down syndrome] and then there will be less infrastructure, and when there is less infrastructure, the more people will want to test. [...] Yes, and people are going to feel obligated to do the test [NIPT]. [...] You are then being forced like 'You can test it and then you will have an abortion' (\#42, high-risk woman)

In contrast, another participant did not think that NIPT would create a 'perfect' society with few or no children with Down syndrome or other disabilities, as exemplified by the following quote:

'Yes, you have people that say 'Then you are only going to have perfect children'. Well, that doesn't happen. Perfect children don't exist. You will always have people that do not choose to have this test or children that become handicapped through an accident or something else' (\#38, high-risk woman)

\section{Widening the scope of prenatal testing: where do you draw the} line?

When participants were asked what they thought about using NIPT to test for other disorders, alongside trisomies 21,13 and 18, they gave mixed reactions. Participants mentioned that for some people, for example, people with a genetic disease in the family, it could be beneficial to test for certain disorders early in pregnancy. Moreover, some participants thought that it was good to use NIPT to get to know as much as possible about your baby and there should be no limit to what can be tested.

'Many times you hear about people that are struggling for a very long time to figure out what is wrong with their child. So why would you limit testing for different disorders? Why would you want to know if a child has Down syndrome, but not whether it has other severe abnormalities?' (\#38, high-risk woman)

On the other hand, some participants were convinced that you should let nature run its course and that you should not want to control everything. The participants argued that you should not strive for a perfect society without any disabilities and therefore they were against screening for many different disorders. Participants also feared a so-called 'slippery slope', which could lead to people starting to test for minor abnormalities, gender or for cosmetic traits like blond hair and blue eyes. They were convinced that a line should be drawn with regard to the extension of NIPT, but they also acknowledged the difficulties associated with this.

'Yes, but on the other hand I think, where do you draw the line? What are you going to test for? Whether he has a cleft lip, a crooked nose? So I wonder, playing the devil's advocate, sure I think it's great to test [...] but where do you draw a line?' (\#14, low-risk woman) 
When asked what kind of disorders they thought it was acceptable to test for, participants felt that the severity of a disorder, the viability of the child and the quality of life were important aspects in deciding whether to test for a certain disorder or not. They mentioned that they would want to have the possibility of testing for severe or fatal disorders that could lead to the early death of the child or a very low quality of life.

'I think that if you could test for disorders where the life expectancy is really low or where you know for sure that children will have a really miserable life, a lot of pain. Yes, then I think that's good.' (\#08, lowrisk woman)

However, participants did expect quality of life and severity of a disorder to be difficult to determine in advance. They argued that even though it might be possible to determine whether an unborn child has a severe disorder, a prenatal test like NIPT cannot predict its severity or the quality of life of the child. Moreover, participants stated that quality of life is a relatively subjective concept and differs per person, which all makes it very difficult to decide whether to test and to continue with the pregnancy or not. Nevertheless, many participants felt that women should be able to make their own decision about what to test for and what not to test for.

'Then you can say 'Where is the limit?' I can't tell, but I think that's something I'd want women to have a choice in. One woman says 'One year life expectancy, I'll take it', while another says 'No thank you' $[\ldots]$ (\#02, low-risk woman)

In the end, most participants agreed that if it would be possible to test for more diseases, there should be a fixed set of disorders for which one could be tested for. They thought that it should not be possible to tick boxes for whether a disease is tested or not, as this is too complex and stressful for women to decide about.

'I think it will cause a pregnant woman too much stress, when she has to decide what should be tested for. You are so happy that you are pregnant, but then you have to think about which one of the 100 diseases.' (\#37, low-risk woman)

\section{DISCUSSION}

Participants felt that the current prenatal screening for trisomies using the combined test has a number of disadvantages that create a high barrier for participation, like the uncertainty of results and the risk of miscarriage from follow-up diagnostic testing. In The Netherlands, these aspects have indeed been shown to be reasons for declining prenatal screening. ${ }^{6,21}$ Participants were therefore very positive about NIPT, as they thought that the characteristics of the test, that is, the increased accuracy, reduced chance of requiring follow-up testing and earlier performance could diminish the disadvantages of prenatal screening and thus lower the barrier for participation. These findings are in line with other studies where positive attitudes and high interest in NIPT were found among pregnant women. ${ }^{9,11,14}$ However, participants in this study realised that the relatively low barrier for the use of NIPT could also have a downside, as it could lead to women feeling less justified to decline this test and thereby jeopardising informed decision-making. This was also shown in a qualitative study by Lewis et $a l^{11}$ in the UK where participants expressed a concern that women might feel pressured to test when a risk-free blood test is available and offered by trusted healthcare professionals. Moreover, another UK study has shown that healthcare professionals think that giving written consent and performing counselling and testing on different days is less important for NIPT than for invasive diagnosis. ${ }^{22}$ These findings support concerns around the potential uncritical use or routinisation of NIPT, which were expressed by some participants. Several participants also feared that the introduction of NIPT could eventually lead to less support for and acceptance of people with disabilities, as fewer disabled children will be born. Although this fear is shared by ethicists, they, as well as some of the participants, also argued that there will always be disabled children as some women will still decline NIPT or decide to continue their pregnancy even if this test identifies a disorder. ${ }^{23}$ Studies performed after the introduction of the national screening program for Down syndrome in The Netherlands in 2007, showed that women at that time were also wary of effects like routinisation, pressure to test and less acceptance of people with disabities. ${ }^{24,25}$ This may imply that these are recurrent concerns that are related to prenatal screening in general. However, the difference with NIPT is that it is likely that more people who currently decline prenatal screening, will now have NIPT and consequently be facing decisions regarding termination of pregnancy where they would not have previously. This might exacerbate the concern that fewer children with Down syndrome will be born. As NIPT might also be more vulnerable to routinisation effects, empirical studies are required to get more insight into the uptake and impact of this test. In California, where NIPT has been offered since 2012, the actual uptake of NIPT, for example, turned out to be lower $(40 \%)^{26}$ than the overall hypothetical interest expressed in other studies ( $\sim 72-82 \%) .{ }^{9,27}$ Moreover, when NIPT was offered to high-risk women, it resulted in fewer women declining follow-up testing. $^{26}$

Participants thought that testing for more types of disorders with NIPT was a development that could be beneficial for severe genetic disorders that are associated with premature death and low quality of life. Other studies also showed that these aspects are widely used justifications for testing, and also termination of pregnancy. ${ }^{28}$ Participants, however, felt that there should be a limit to what is allowed to be tested with NIPT, to prevent people from testing for minor abnormalities, gender or cosmetic traits. These findings are similar to results from a recent UK study by Farrimond et al. ${ }^{29}$

This is one of the first qualitative studies in The Netherlands to explore the attitudes of pregnant women about NIPT and their views on widening the scope of prenatal screening with the use of NIPT. Moreover, this study also included male partners as they have an important role in the decision-making process for prenatal screening or diagnosis. No striking differences in the attitudes between the women and men were found in this study. However, as different qualitative research methods were used, that is, focus groups and online focus groups, the conclusion that women and men have similar attitudes towards NIPT should be drawn with some caution. The study sample consisted of both low-risk and high-risk women who had a variety of experiences in prenatal testing. However, participants were predominantly white and higher educated and as these variables are associated with higher levels of interest in NIPT, ${ }^{9}$ it could be that this influenced the results in favour of NIPT. Moreover, participants were interviewed late in their pregnancy which might have affected the results of this study. It is also likely that participants have taken part in this study because of an interest in prenatal screening and this might have created a selection bias.

In conclusion, the findings suggest that NIPT might make the decision for prenatal screening more meaningful. Whereas now decision-making about prenatal screening is partly influenced by fear of miscarriage or uncertainty of test results, introduction of NIPT will 
allow women and their partners to make a decision on prenatal screening that is based solely on their wish to know or not know whether their child is affected. This is an important observation given the widely accepted view that the aim of prenatal screening for foetal abnormalities is to provide meaningful options for reproductive choice. ${ }^{30}$ Pressure to test and routinisation of NIPT, however, could have the opposite effect by undermining reproductive autonomy. This implies that safeguards ensuring voluntary participation on the basis of informed decision-making will be of utmost importance. If in a future scenario NIPT is to be used to test for a wider range of disorders, informed decision-making may be further challenged by information overload and decisional complexity. It has been suggested that this may require a new approach to informed choice, based on generic information about categories of outcomes rather than about specific disorders. ${ }^{7}$ For a successful implementation of NIPT in The Netherlands, perspectives of pregnant women and partners should be taken into account and additional research is needed among a more representative study population to generalise the findings of this study.

\section{CONFLICT OF INTEREST}

The authors declare no conflict of interest.

\section{ACKNOWLEDGEMENTS}

The authors wish to thank the women and men who participated in the study. Janneke Gitsels is acknowledged for helping in the recruitment of participants. The study was funded by The Netherlands Organisation for Health Research and Development (ZonMw) as part of the Regional Perinatal Network Northwest Netherlands, and is also part of the research programme of the CSG Centre for Society and the Life Sciences, funded by The Netherlands Genomics Initiative.

1 Lo YMD: Non-invasive prenatal diagnosis by massively parallel sequencing of materna plasma DNA. Open Biol 2012; 2: 120086.

2 Wright CF, Burton $\mathrm{H}$ : The use of cell-free fetal nucleic acids in maternal blood for non-invasive prenatal diagnosis. Hum Reprod Update 2009; 15: 139-151.

3 Morain S, Greene MF, Mello MM: A new era in noninvasive prenatal testing. $N$ Engl $\mathrm{J}$ Med 2013; 369: 499-501.

4 Lo YMD, Chan KCA, Sun $\mathrm{H}$ et al: Plasma DNA sequencing reveals the genome-wide genetic and mutational profile of the fetus. Sci Trans/ Med 2010; 2: 61ra91.

5 Greely HT: Get ready for the flood of fetal gene screening. Nature 2011; 469 289-291.

6 Van den Berg M, Timmermans DR, Kleinveld JH, Garcia E, Van Vugt JM, Van der Wal G Accepting or declining the offer of prenatal screening for congenital defects: test uptake and women's reasons. Prenat Diagn 2005; 25: 84-90.

7 De Jong A, Dondorp WJ, De Die-Smulders CEM, Frints SGM, De Wert GMWR: Non-invasive prenatal testing: ethical issues explored. Eur J Hum Genet 2010; 18: 272-277.
8 Hall A, Bostanci A, John S: Ethical, legal and social issues arising form cell-free fetal DNA technologies. Appendix III to the report: Cell-free fetal nucleic acids for noninvasive prenatal diagnosis, Cambridge, 2009, pp 1-45.

9 Tischler R, Hudgins L, Blumenfeld YJ, Greely HT, Ormond KE: Noninvasive prenatal diagnosis: pregnant women's interest and expected uptake. Prenat Diagn 2011; 31: 1292-1299.

10 Yotsumoto J, Sekizawa A, Koide $\mathrm{K}$ et al: Attitudes toward non-invasive prenatal diagnosis among pregnant women and health professionals in Japan. Prenat Diagn 2012; 32: 674-679.

11 Lewis C, Silcock C, Chitty LS: Non-invasive prenatal testing for Down's syndrome: pregnant women's views and likely uptake. Publ Health Genom 2013; 16: 223-232.

12 Hill M, Fisher J, Chitty LS, Morris S: Women's and health professionals' preferences for prenatal tests for Down syndrome: a discrete choice experiment to contrast noninvasive prenatal diagnosis with current invasive tests. Genet Med 2012; 14: 905-913.

13 Draper J: 'It's the first scientific evidence': men's experience of pregnancy confirmation. J Adv Nurs 2002; 39: 563-570.

14 Kooij L, Tymstra T, Van den Berg P: The attitude of women toward current and future possibilities of diagnostic testing in maternal blood using fetal DNA. Prenat Diagn 2009; 29: 164-168.

15 Schielen PCJI: Quality control parameters of Dutch Down's syndrome screening laboratories, Bilthoven, 2010. RIVM Report 230083003/2012.

16 Ekelund CK, Petersen OB, Skibsted L, Kjaergaard S, Vogel I, Tabor A: First-trimester screening for trisomy 21 in Denmark: implications for detection and birth rates of trisomy 18 and trisomy 13. Ultrasound Obstet Gynecol 2011; 38: 140-144.

17 Blondel B, Kermarrec M: Enquête nationale périnatale 2010. Les naissances en 2010 et leur évolution depuis 2003. INSERM: ParisReport, 2011; p 117.

18 Krueger RA, Casey MA: Focus groups. A practical Guide For Applied Research. Sage Publications: Thousand Oaks, 2000.

19 Tates K, Zwaanswijk M, Otten R et al: Online focus groups as a tool to collect data in hard-to-include populations: examples from paediatric oncology. BMC Med Res Methodol 2009; 9: 15.

20 Pope C, Ziebland S, Mays N: Qualitative research in health care. Analysing qualitative data. BMJ 2000; 320: 114-116.

21 Bakker M, Birnie E, Pajkrt E, Bilardo CM, Snijders RJM: Low uptake of the combined test in The Netherlands-which factors contribute? Prenat Diagn 2012; 32 $1305-1312$.

22 Van den Heuvel A, Chitty L, Dormandy $E$ et al: Will the introduction of non-invasive prenatal diagnostic testing erode informed choices? An experimental study of health care professionals. Patient Educ Couns 2010; 78: 24-28.

23 Cole R, Jones G: Testing times: do new prenatal tests signal the end of Down syndrome? N Z Med J 2013; 126: 96-102.

24 Garcia E, Timmermans DRM, Van Leeuwen E: The impact of ethical beliefs on decisions about prenatal screening tests: searching for justification. Soc Sci Med 2008; 66: 753-764.

25 Garcia E, Timmermans DRM, Van Leeuwen E: Parental duties and prenatal screening does an offer of prenatal screening lead women to believe that they are morally compelled to test? Midwifery 2012; 28: 837-843.

26 Chetty S, Garabedian MJ, Norton ME: Uptake of noninvasive prenatal testing (NIPT) in women following positive aneuploidy screening. Prenat Diagn 2013; 33: 542-546.

27 Verweij EJ, Oepkes D, De Boer MA: Changing attitudes towards termination of pregnancy for trisomy 21 with non-invasive prenatal trisomy testing: a populationbased study in Dutch pregnant women. Prenat Diagn 2013; 33: 397-399.

28 Hewison J: Social and ethnic differences in attitudes and consent to prenatal testing. ESRC Full Research Report, 2005. L21825: ESRC: Swindon.

29 Farrimond HR, Kelly SE: Public viewpoints on new non-invasive prenatal genetic tests Public Underst Sci 2013; 22: 730-744.

30 De Jong A, Dondorp WJ, Frints SGM, De Die-Smulders CEM, De Wert GMWR: Advances in prenatal screening: the ethical dimension. Nat Rev Genet 2011; 12: 657-663. 